\title{
Synthetic Musk Fragrances in Lake Michigan
}

\author{
Aaron M. Peck and Keri C. Hornbuckle*
}

Dept. Civil and Environmental Engineering, University of Iowa, Iowa City, IA

8 Pages

14 Figures 


\section{TIC and Mass Spectra of HHCB Technical Mixture}

The HHCB standard used in this work was a technical mixture containing about $74 \% \mathrm{HHCB}$. The figures below show the TIC (Figures SI-1 and SI-2) for the mixture and mass spectra for 10 peaks in this chromatogram (Figures SI-3 - SI-12). The major ion in the mass spectra for peaks $\mathrm{A}, \mathrm{B}$, and $\mathrm{J}$ is 229 . The major ion in the remaining peaks (C, D, E, F, G, H, and I) is 243 . The coeluting peaks ( $C$ and $D)$ have identical mass spectra; these peaks are distinctly separated with injection of lower mass using SIM as shown in Figure 2 in the text.

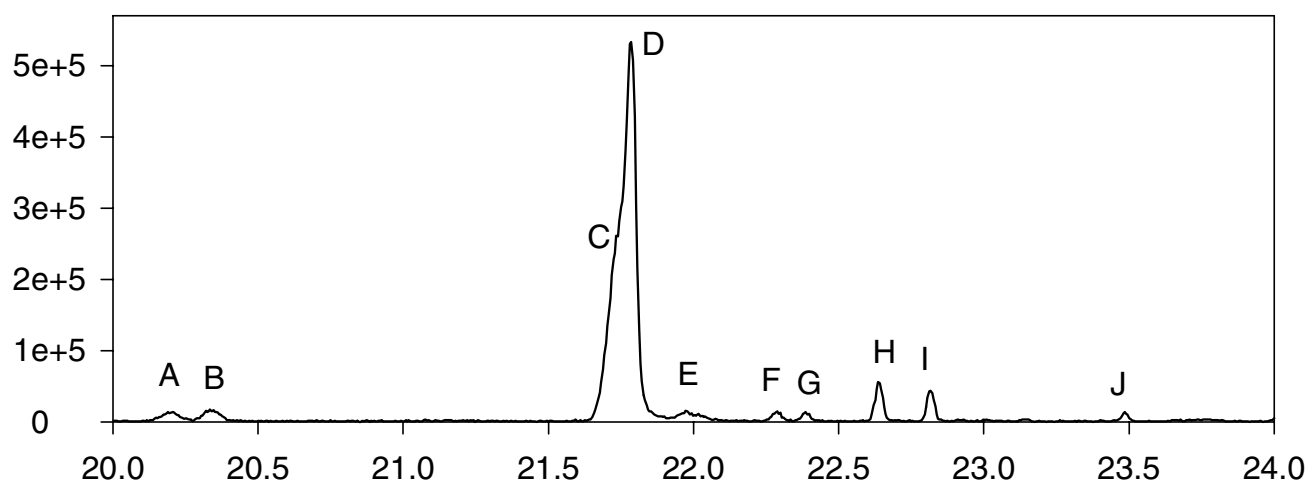

Figure SI-1. TIC of HHCB technical standard ( 74\% HHCB).

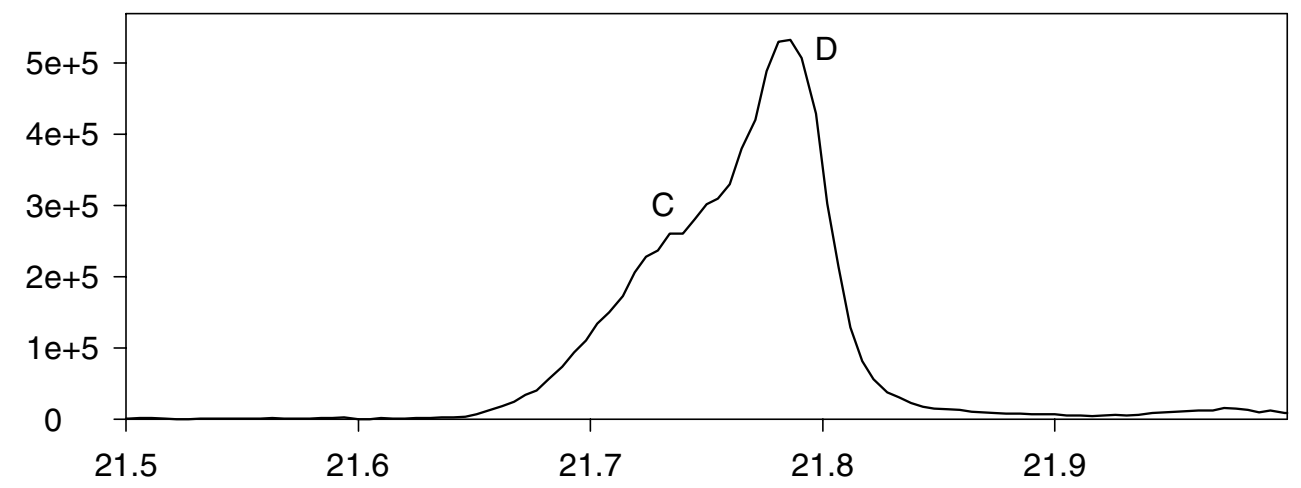

Figure SI-2. TIC of HHCB (D) and coeluting peak (C). 


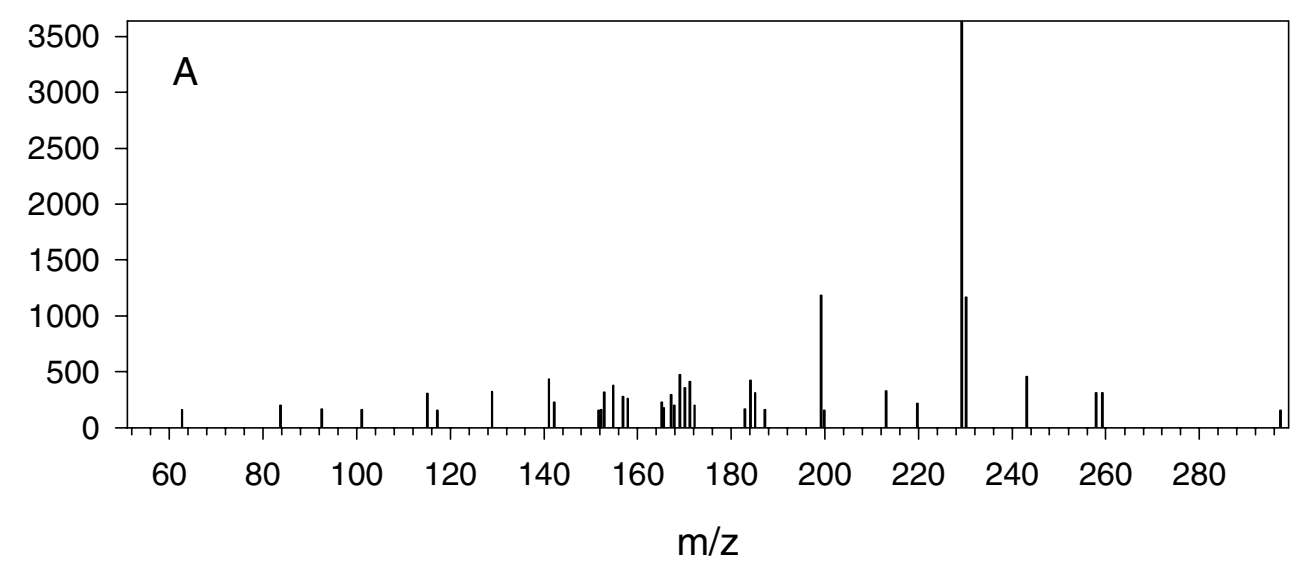

Figure SI-3. Mass spectrum of peak A (20.20 min).

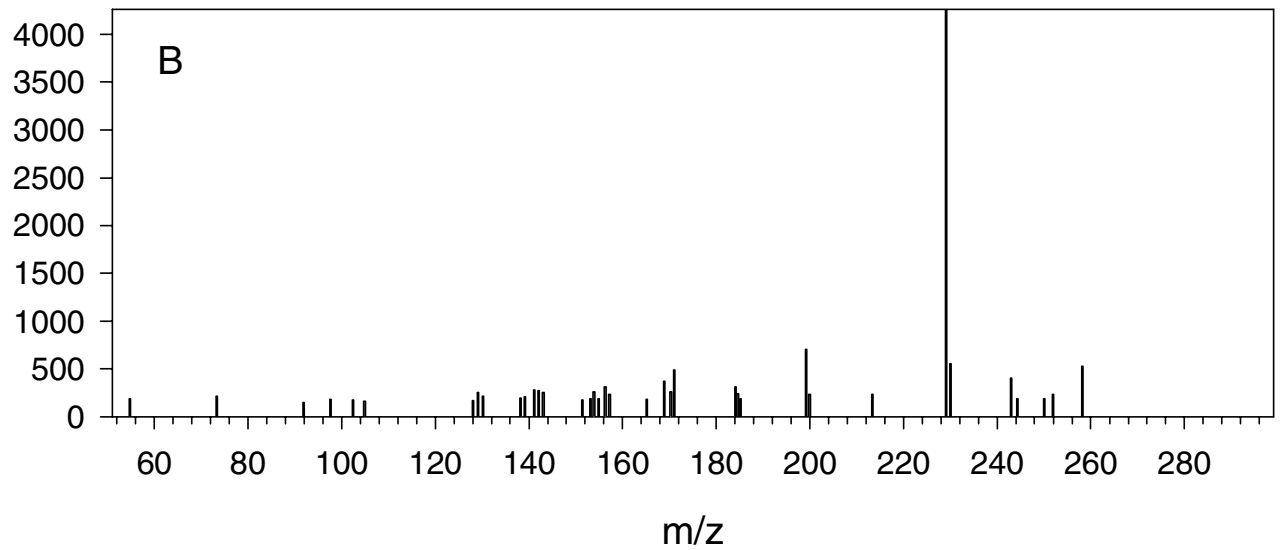

Figure SI-4. Mass spectrum of peak B (20.33 min).

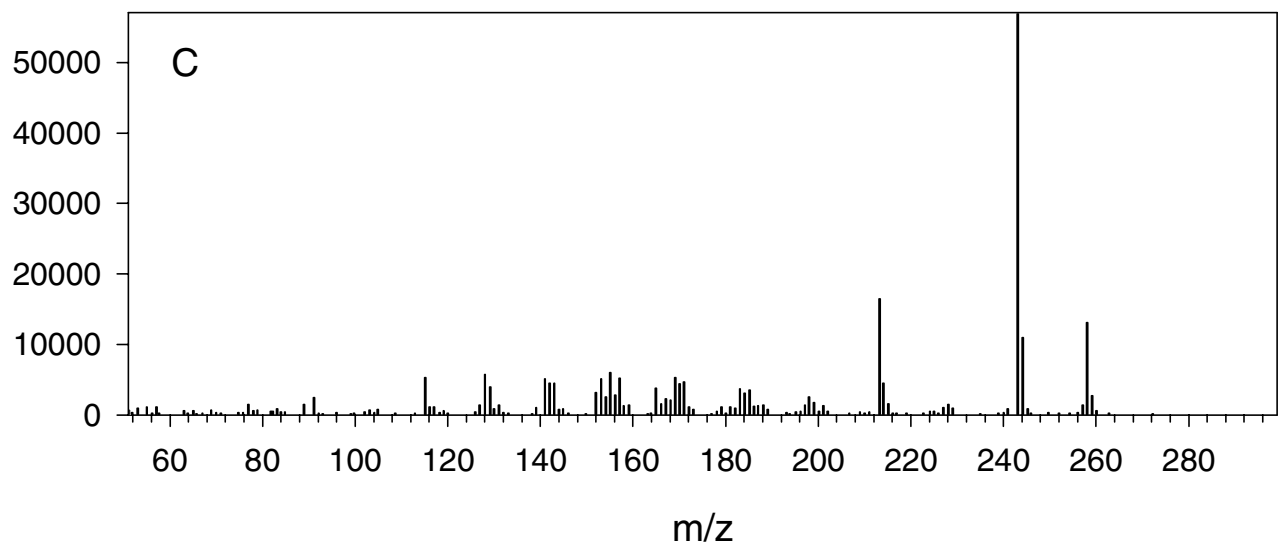

Figure SI-5. Mass spectrum of peak C (21.73 min). 


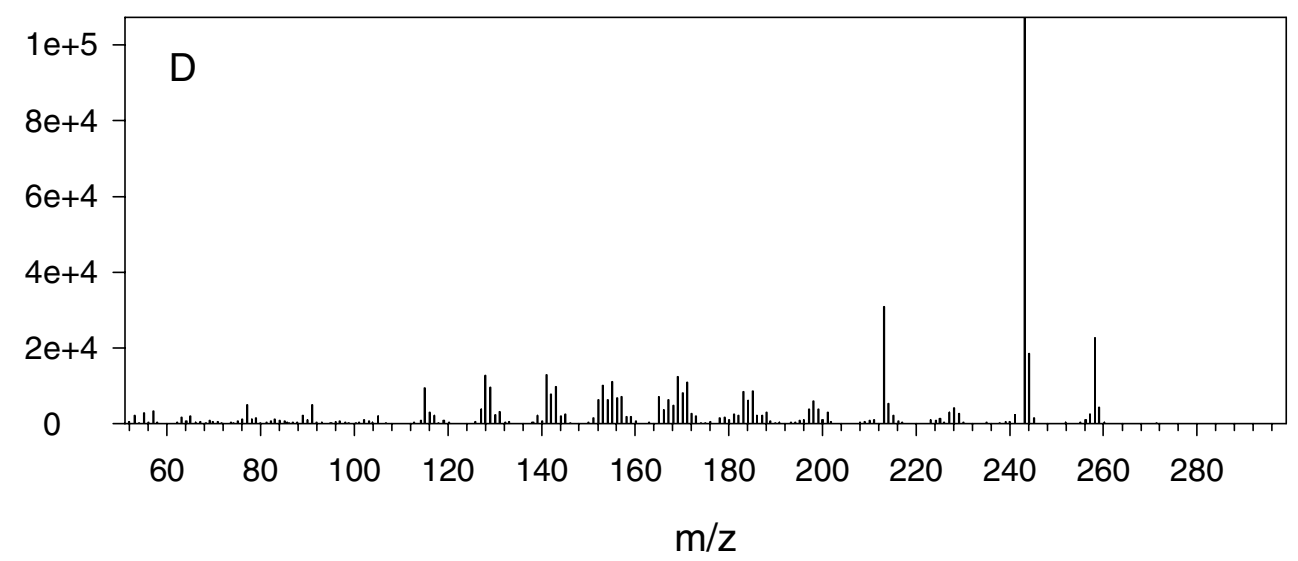

Figure SI-6. Mass spectrum of peak D (21.79 min).

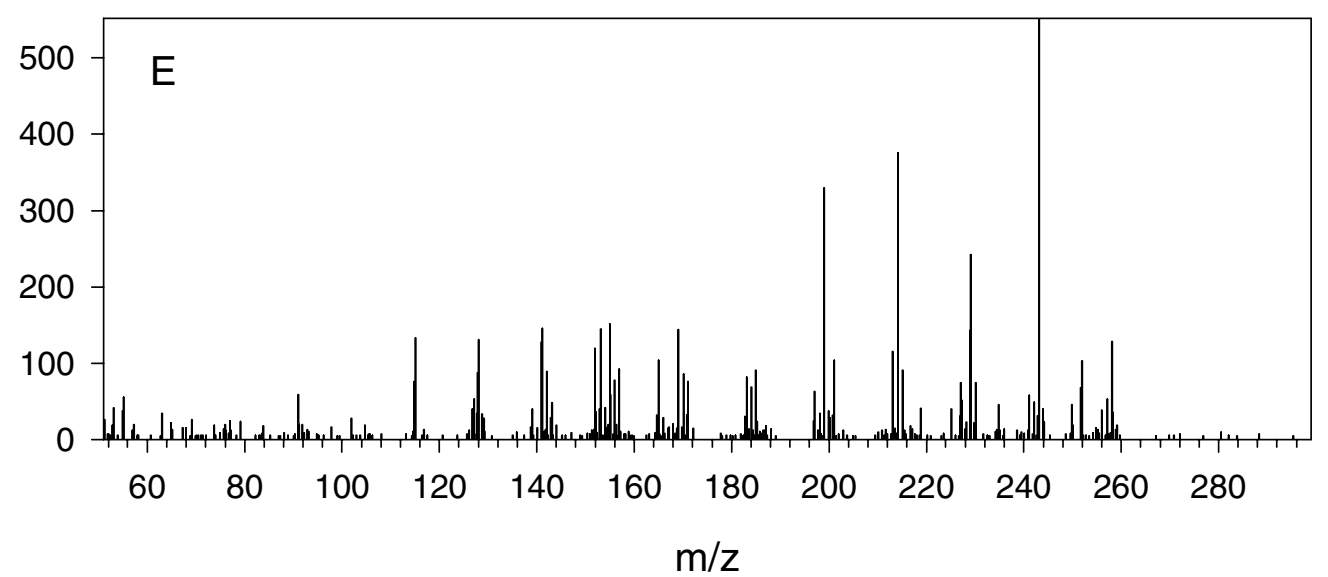

Figure SI-7. Mass spectrum of peak E (22.01 min).

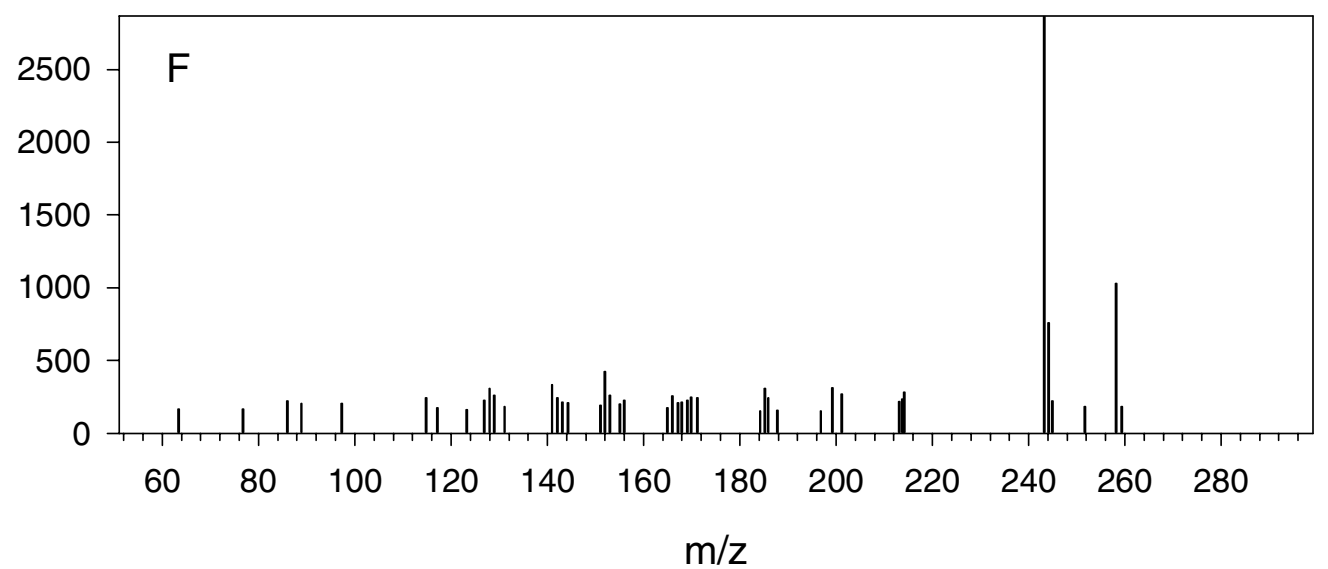

Figure SI-8. Mass spectrum of peak F (22.29 min). 


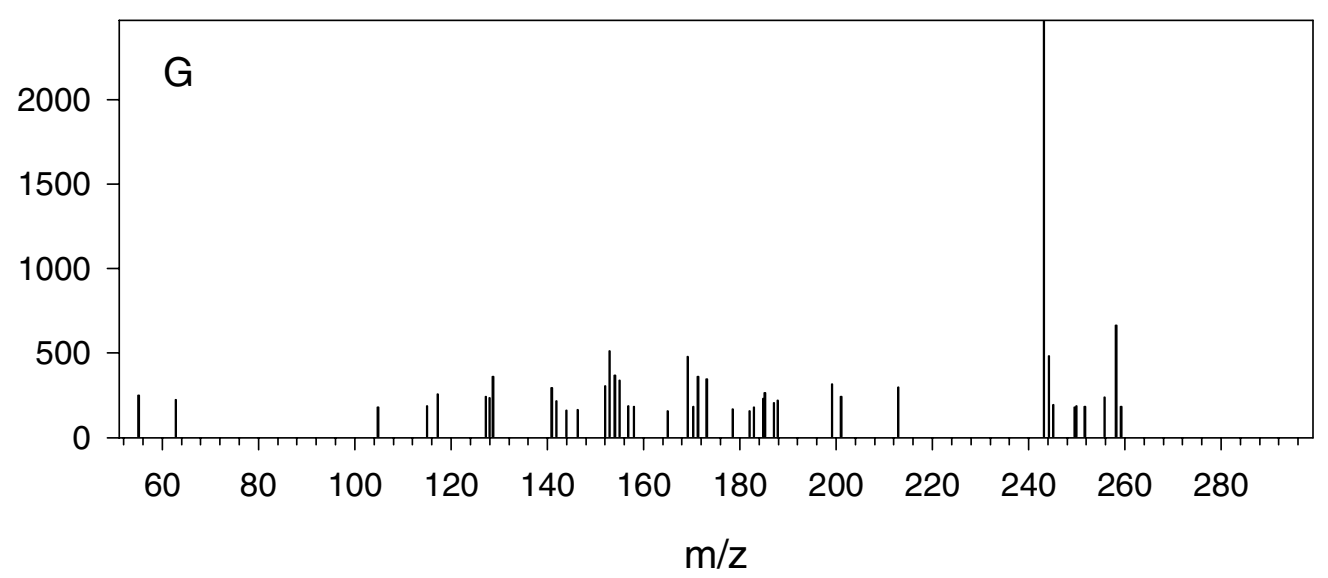

Figure SI-9. Mass spectrum of peak G (22.39 min).

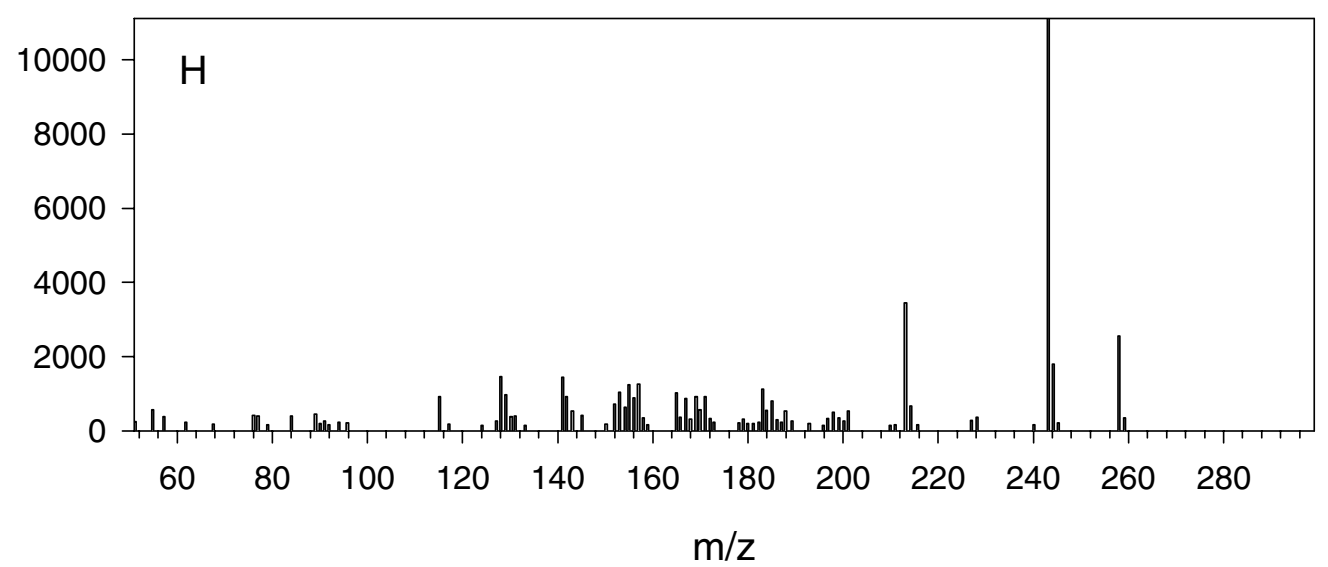

Figure SI-10. Mass spectrum of peak H (22.64 min).

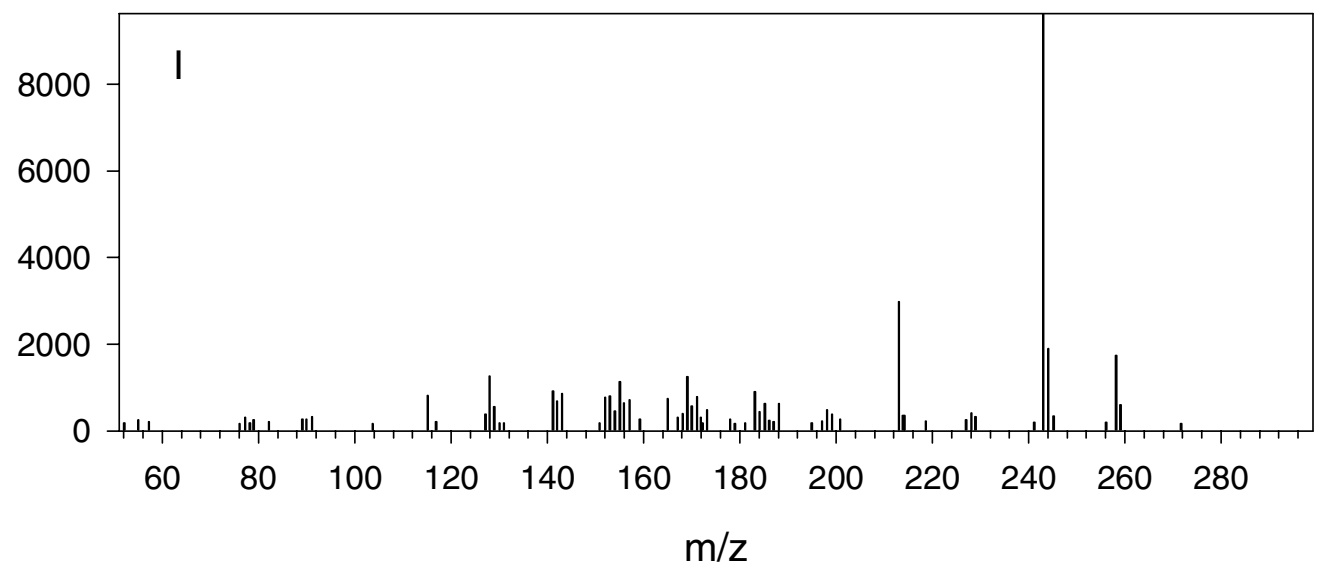

Figure SI-11. Mass spectrum of peak I (22.82 min). 


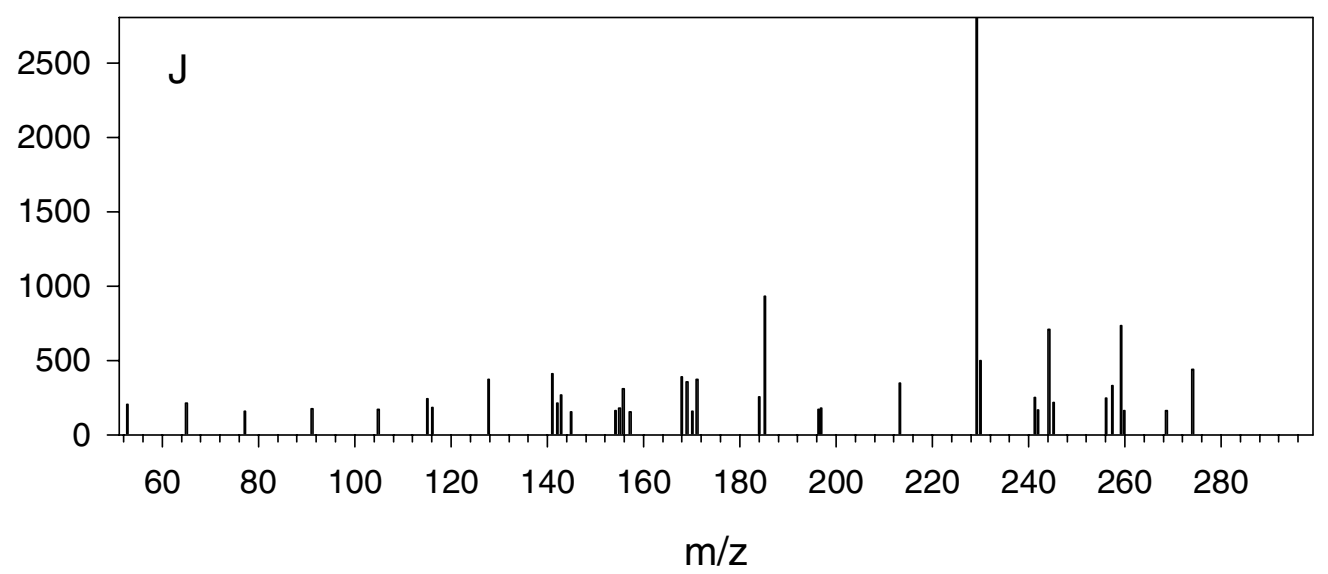

Figure SI-12. Mass spectrum of peak J (23.49 min). 


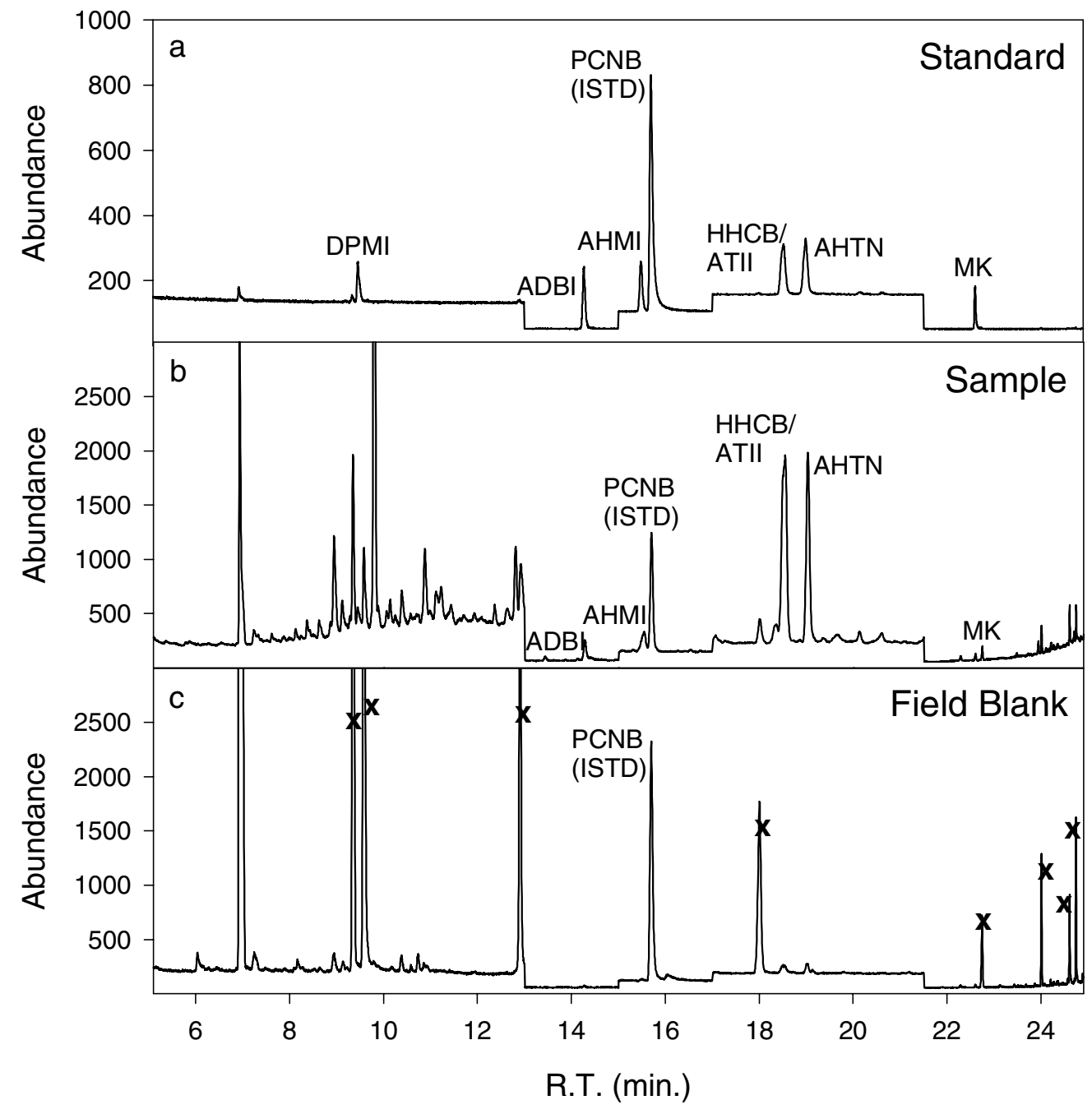

Figure SI-13. Example chromatograms. A) Calibration standard. B) Gas-phase sample. Unlabeled peaks are unidentified. C) XAD air field blank. ' $\mathrm{X}$ ' marks unidentified peaks. Although a peak at about 11 minutes coelutes with DPMI, the DPMI confirmation ion is not present in the field blanks or sample. 

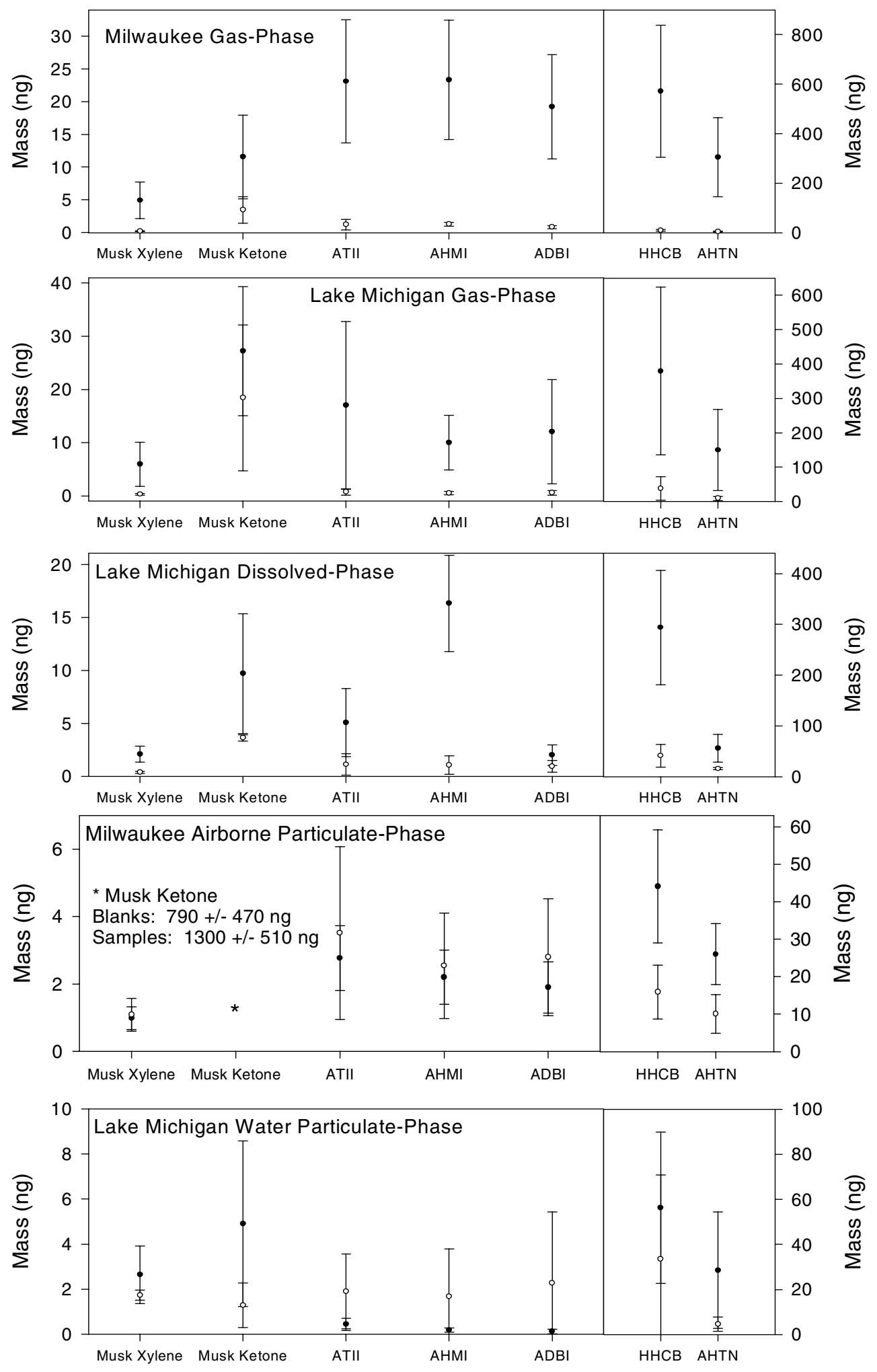

Figure SI-14. 95\% confidence intervals of sample and field blank masses. Closed circles are the average sample masses and open circles are the average field blank masses. The error bars show the $95 \%$ confidence interval. 